\title{
Quantitative Analysis of Spinal Canal Areas in the Lumbar Spine: An Imaging Informatics and Machine Learning Study
}

(D) B. Gaonkar, (DD. Villaroman, (D). Beckett, (DC. Ahn, (D). Attiah, (DD. Babayan, (D).P. Villablanca, (D) N. Salamon, (D) A. Bui, and (D) L. Macyszyn

\section{ABSTRACT}

BACKGROUND AND PURPOSE: Quantitative imaging biomarkers have not been established for the diagnosis of spinal canal stenosis. This work aimed to lay the groundwork to establish such biomarkers by leveraging the developments in machine learning and medical imaging informatics.

MATERIALS AND METHODS: Machine learning algorithms were trained to segment lumbar spinal canal areas on axial views and intervertebral discs on sagittal views of lumbar MRIs. These were used to measure spinal canal areas at each lumbar level (L1 through L5). Machine-generated delineations were compared with 2 sets of human-generated delineations to validate the proposed techniques. Then, we use these machine learning methods to delineate and measure lumbar spinal canal areas in a normative cohort and to analyze their variation with respect to age, sex, and height using a variable-intercept mixed model.

RESULTS: We established that machine-generated delineations are comparable with human-generated segmentations. Spinal canal areas as measured by machine are statistically significantly correlated with height $(P<.05)$ but not with age or sex.

CONCLUSIONS: Our machine learning methodology demonstrates that this important anatomic structure can be accurately detected and quantitatively measured without human input in a manner comparable with that of human raters. Anatomic deviations measured against the normative model established here could be used to flag spinal stenosis in the future.

ABBREVIATIONS: $\mathrm{CPT}=$ Current Procedural Terminology; ERT $=$ ensemble of regression trees; ICD-9 = International Classification of Diseases; $\mathrm{ML}=$ machine learning; MRN = medical record number; $S V M=$ support vector machine

$\mathbf{S}$ pinal cord or nerve root compression due to narrowing of the spinal canal is thought to underlie the disorders of lumbar radiculopathy and myelopathy, both major causes of morbidity and disability ${ }^{1,2}$ in the United States. Patient screening includes radiologic evaluation of the central canal of the spine using MR imaging alongside labeling of stenosis as none, mild, moderate, or severe. These labels drive risky and often expensive treatment and surgical decisions. Yet, MR imaging-based labeling is known to be highly subjective and shows substantial interrater variability. ${ }^{3-5}$ It is necessary to develop objective diagnostic and treatment criteria $^{6}$ to improve treatment.

Received September 19, 2018; accepted after revision July 3, 2019

From the Departments of Neurosurgery (B.G., D.V., J.B., C.A., M.A., D.B., L.M.) and Radiology (J.P.V., N.S., A.B., L.M.), University of California, Los Angeles, Los Angeles, California.

Please address correspondence to Bilwaj Gaonkar, PhD, Department of Neurosurgery, 300 Stein Plaza, Ste 554E, Los Angeles, CA, 90095; e-mail: bilwaj@gmail.com; @bilwaj

Indicates article with supplemental on-line appendix and tables.

Indicates article with supplemental on-line photo.

http://dx.doi.org/10.3174/ajnr.A6174
Canal stenosis by definition is a reduction in the area of the spinal canal. The percentage reduction in canal area compared with a demographically matched control signifies the degree of stenosis. Yet, computing the percentage reduction requires that one can consistently and accurately delineate spinal canals on MR imaging and that one has knowledge of the variation of canal areas in asymptomatic individuals over a wide demography. Our study presents work in both directions by proposing and validating a machine learning $(\mathrm{ML})$ method to automatically delineate spinal canals on axial MR imaging using the validated ML method in conjunction with a large clinical data base to establish a variable-intercept mixed linear model of variation of spinal canal areas.

While computational methods to segment anatomic ROIs have been published in the literature ${ }^{7-10}$ and used to segment several regions in the spine, ${ }^{8,9,11-15}$ we focused on the spinal canal. We established an ML technique to delineate spinal canals on axial MR imaging and to measure their areas at lumbar levels. Subsequently, we established a linear model linking these areas to age, sex, and height using data from 1755 asymptomatic individuals. 


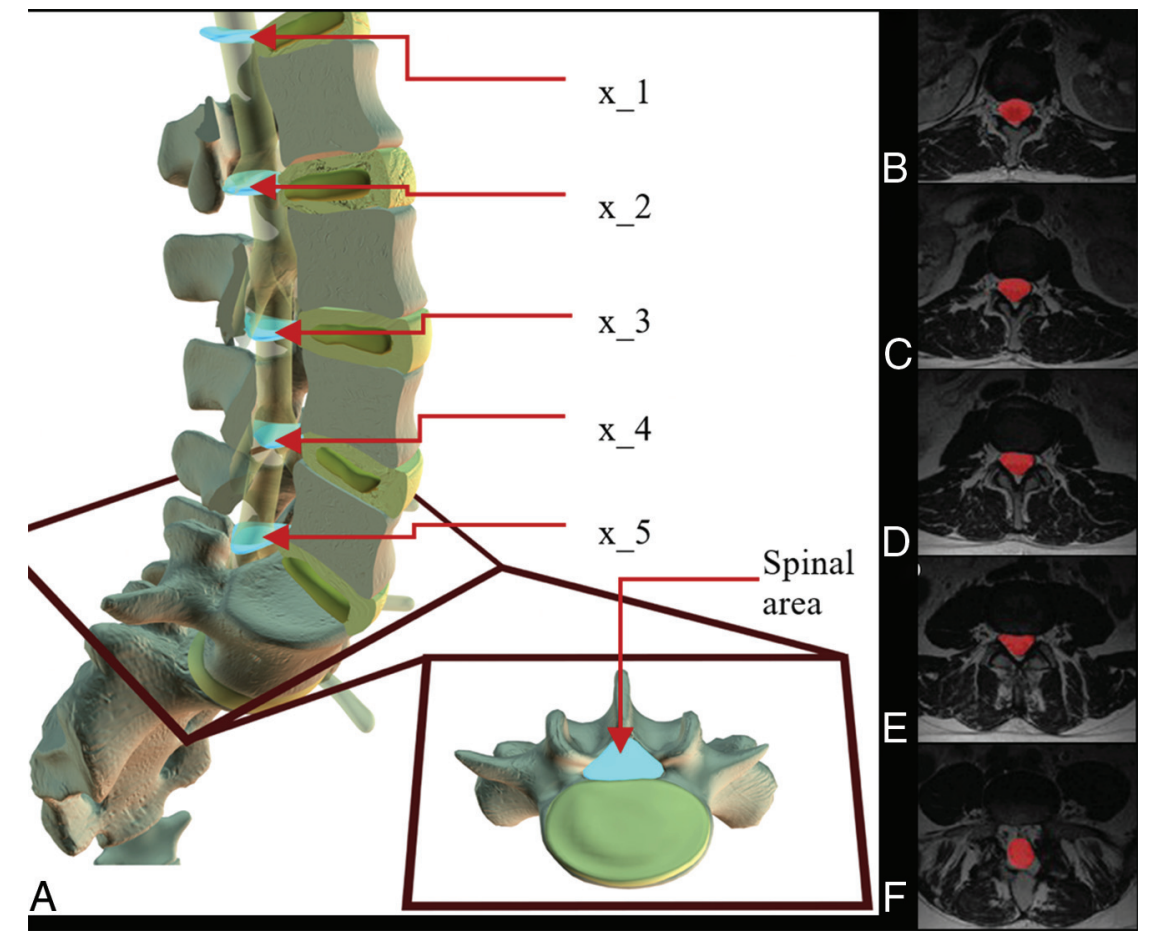

FIG 1. Variation of spinal canal area with level. $A$, This $3 D$ model represents a generic lumbar spine where light blue objects represent an area of the central canal at each lumbar level at the midsection of a disc. The square frame (red) zooms in on the intervertebral disc (yellow) below L5 to give an axial view of where the central canal area (light blue) is located. In a randomly selected T2MR imaging, each picture in this series $B-F$ depicts 1 section of spinal cord segmentation (red) from each level. Tissues within the canal but outside the thecal sac are not segmented.

and anonymized the images corresponding to each accession number. The On-line Appendix presents further details of our data collection.

\section{MR Imaging Sequences}

Axial T2 MR imaging was used for canal segmentation. Resolutions in the axial plane varied between $0.27 \times$ $0.27 \mathrm{~mm}$ per pixel to $1.5 \times 1.5 \mathrm{~mm}$ Resolutions were perpendicular to the axial plane and ranged between 1 and $10 \mathrm{~mm}$. The mean resolution was $0.53 \times 0.53 \mathrm{~mm}$ in the axial plane and $5.13 \mathrm{~mm}$ in the perpendicular direction. Corresponding SDs were $0.125 \times$ 0.125 and $0.5 \mathrm{~mm}$, respectively. The mean TRs and TEs varied as TR $=$ $3756 \pm 738 \mathrm{~ms}$ and $\mathrm{TE}=107 \pm$ $12 \mathrm{~ms}$. Corresponding sagittal images used for disc segmentation had resolutions between $0.5 \times 0.5$ and $2 \times 2 \mathrm{~mm}$ per pixel in the sagittal plane and $1-5$ $\mathrm{mm}$ perpendicular to the sagittal plane.

\section{Preprocessing}

Preprocessing involved nonparametric bias correction, linear histogram matching to a common template, and

The study was executed in 4 steps:

1. Creating a large data base of lumbar MRI studies.

2. Training and validating ML models for delineating canals and measuring their areas, using subsets of data extracted from the database and manually segmented by experts.

3. Using the ML models to measure canal areas in asymptomatic individuals with MRIs.

4. Using these measurements to establish a linear model linking lumbar spinal canal areas to age, sex, and height.

Many in the radiology community agree that there are numerous advantages of standardized reports, ${ }^{16}$ and this study aims to usher in a quantitative era for radiologic interpretation and reporting for lumbar spinal stenosis.

\section{MATERIALS AND METHODS}

\section{Institutional Review Board Statement}

This study was conducted according to the rules and regulations of our institution and approved by the institutional review board (institutional review board No. 16-000196).

\section{Data Collection for Machine Learning}

We queried the PACS of our institution for individuals who had undergone any spine imaging using the corresponding Current Procedural Terminology $(\mathrm{CPT})^{17}$ codes (On-line Table 1). This query yielded 39,295 unique medical record numbers (MRNs) and corresponding accession numbers. We extracted intensity normalization to the $0-1$ range for each $3 \mathrm{D}$ MR image. All scans were oriented into the frame of the template using linear image registration, and resampling was performed in the axial frame to fit each section to a $256 \times 256$ pixel frame.

\section{Training Data Generation by Human Raters}

A subset of 100 axial MR images was randomly chosen from the 39,295 for algorithmic training purposes and archived alongside corresponding sagittal MR images. Physicians segmented spinal canals and discs with the help of students. A student was first trained by an attending physician to identify spinal canal boundaries and delineate them using ITK-SNAP (www.itksnap.org). ${ }^{18}$ The student delineated canals on each section of the 100 axial MRIs and saved the segmentations as NIfTI files. The student also went through the 100 corresponding sagittal MRIs and segmented lumbar discs. The attending physician reviewed each section and corrected the student-generated delineations. The segmented spinal canal region was the area enclosed in the thecal sac, excluding ligaments and structures within the cavity. Segmenting the thecal sac within the spinal canal allows more distinct edges and defines a more clinically relevant area. These scans were used for training the models.

\section{Validation Data Generation}

The process of segmenting spinal canals on axial scans was repeated 2 more times on 109 axial images with different studentphysician pairs, similar to the training data generation. These 


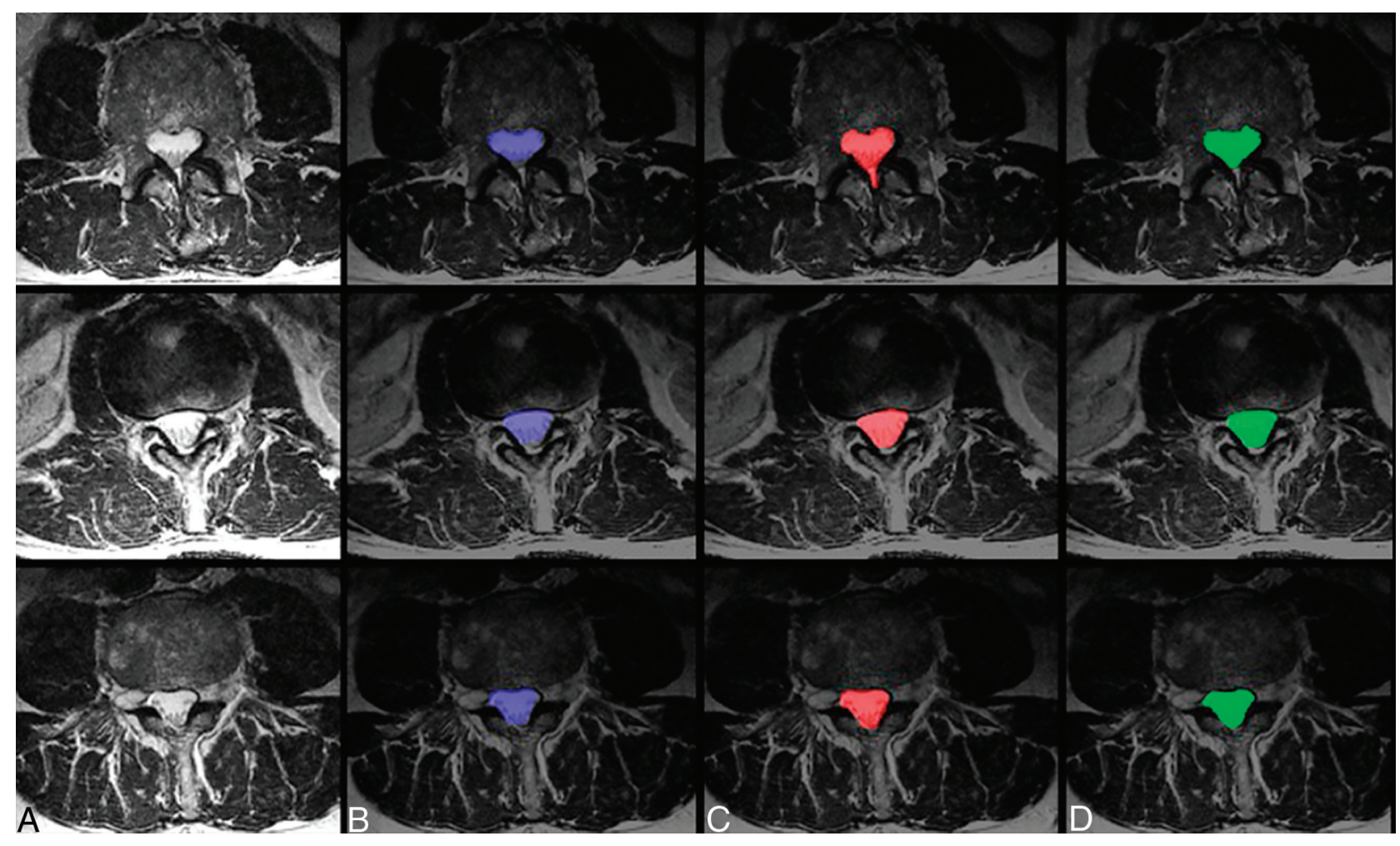

FIG 2. Sample case images of central canal segmentations. Three case images of axial T2 MR imaging $(A)$ randomly selected from the dataset are shown alongside their resulting segmentations (blue) of the spinal canal using the proposed ensemble technique (B), segmentation (red) by manual rater $1(C)$, and segmentation (green) by manual rater $2(D)$.

Table 1: Comparison of automated spinal canal segmentations in a validation dataset of 109 axial MRIs ${ }^{2}$

\begin{tabular}{llccc}
\hline & Centrality & $\begin{array}{c}\text { Auto vs } \\
\text { Rater 1 }\end{array}$ & $\begin{array}{c}\text { Auto vs } \\
\text { Rater 2 }\end{array}$ & $\begin{array}{c}\text { Rater 1 vs } \\
\text { Rater 2 }\end{array}$ \\
\hline Dice ratio & Mean & $0.84 \pm 0.08$ & $0.83 \pm 0.08$ & $0.9 \pm 0.05$ \\
& Median & 0.87 & 0.85 & 0.92 \\
Hausdorff distance $(\mathrm{mm})$ & Mean & $7.89 \pm 9.42$ & $9.41 \pm 11.2$ & $7.90 \pm 9.62$ \\
& Median & 4.59 & 5.64 & 4.66 \\
Average surface distance $(\mathrm{mm})$ & Mean & $0.84 \pm 0.08$ & $0.83 \pm 0.08$ & $0.9 \pm 0.05$ \\
& Median & 0.10 & 0.14 & 0.07 \\
\hline
\end{tabular}

with $\nu=0.05$ and a tree depth set to 2 to predict 68 points, which form the contour of each spinal canal. Both steps were implemented using the DLib 1.8.0 software library (http:// dlib.net/).

\section{Disc Segmentation}

A Deep-U-Net ${ }^{7}$ model (On-line Figure) was trained on the designated

were selected by randomly sampling from the $39,000+$ MRNs containing symptomatic and asymptomatic cases.

\section{Training the Machine Learning Model for Segmentation of the Central Canal}

We used a hybrid machine learning model to execute segmentation of central canals. In the first step, we detected a $25 \times 25$ pixel window containing the canal. An ensemble of support vector machine (SVM)-based object detection systems was trained using histogram-of-oriented gradient ${ }^{19}$ features and the hard-negative mining paradigm to "classify" whether a particular $25 \times 25$ pixel window contained a central canal. The SVMs used were linear SVMs with $\mathrm{C}=10,50,100,150,250,500,1000$. A window classified by $\geq 4$ SVMs as the spinal canal was considered a "positive" detection. The image was cropped along this window and passed on to the second step of segmentation, which was executed using an ensemble of regression trees ${ }^{20}$ (ERT) shape-regression model. We used the ERT
100 sagittal MR images to segment discs and was implemented using the Keras API running on top of TensorFlow 1.3.0, A rectified linear unit was used for convolutional neurons throughout the architecture except for the final output layer, which used sigmoidal activation. We used a fixed learning rate (1e-5) and the Adam optimizer with drop-out (probability of .25) regularization. The loss function used was the negative of the Dice score.

\section{Segmentation Measurements}

We used Dice scores, the Hausdorff distance, and average surface distance metrics. These compared overlaps for automatic spinal canal segmentations with segmentations generated by manual raters and manual raters among themselves.

\section{Data Collection for Analysis of Normative Cohort}

We cross-referenced the 39,295 image accessions with anonymized patient records to eliminate studies associated with the 


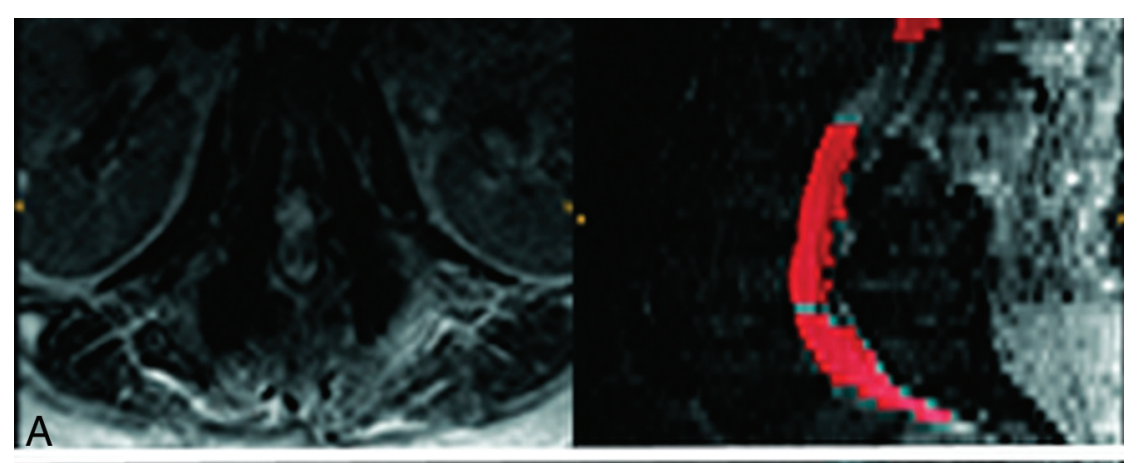

\section{Area Measurements}

We cross-referenced axial MRIs containing segmented canals with their sagittal MRIs containing segmented intervertebral discs to locate slices at each lumbar level in a standard way (Fig 1). At axial slices where the center of a disc was found, we documented canal areas to investigate variation of these areas with respect to age, sex, and height.

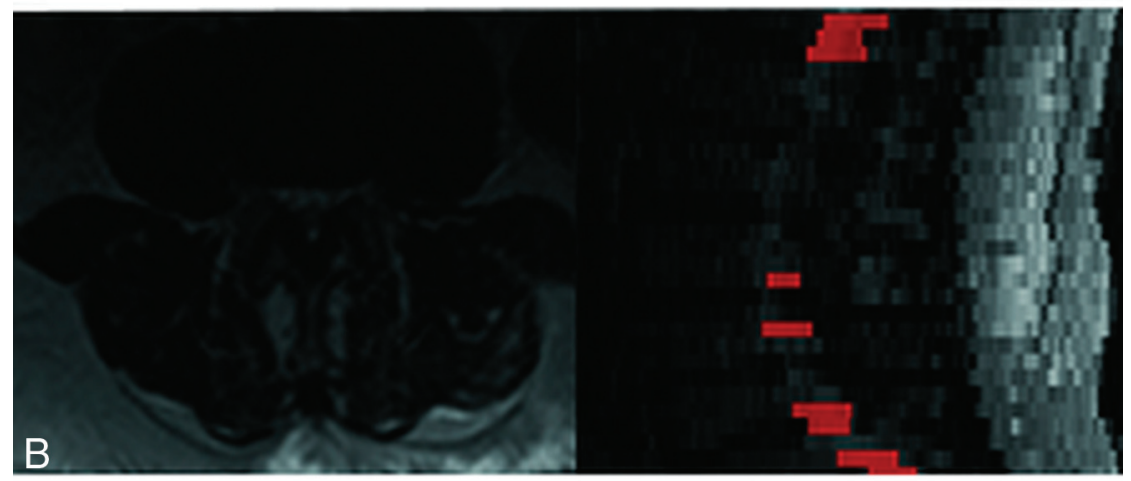

\section{RESULTS}

\section{Segmentation Results}

While central canals may not have a consistent shape (Fig $2 A$ ), machine-generated segmentations were qualitatively comparable with those generated by human experts (Fig 2B-D). Quantitative metrics (Dice score, Hausdorff distance, and average surface distance) for the validation dataset are recorded in Table 1. These metrics indicate that machine-

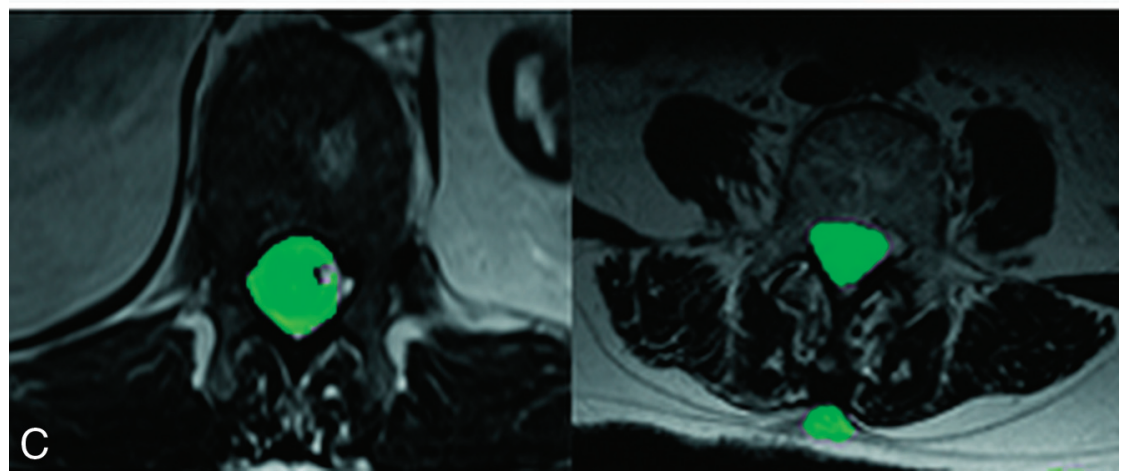

FIG 3. Modes of segmentation failure of the proposed algorithm compared with U-Net results. Two scans using SVM + ERT failed (Dice score $<0.7$ ). The mode of failure was complete lack of segmentation as seen in rows $A$ and $B$, thereby making it easy to eliminate such cases automatically. In contrast $\mathrm{U}$-Net failures are more subtle and can involve under and oversegmentation as shown in row $C$. generated segmentation agrees almost as well with each human expert as the human experts agree among themselves. Disc segmentations generated by machine achieved a Dice overlap of 0.88 with respect to a single human rater on the validation dataset. All discs detected by the human rater were detected by the U-Net, achieving a detection rate of $100 \%$.

\section{Segmentation Modes of Failure}

Figure $3 A,-B$ presents 2 validation set images that had a Dice coefficient $<0.7$. The proposed model seems to fail by nonsegmentation of entire slices. Given that U-Net-generated disc segmenta-

International Classification of Diseases ${ }^{21}$ (ICD-9) codes (listed in On-line Table 2) related to the presence of spinal pathology or symptoms attributable to the spine (eg, sciatica). The filtered dataset contained 3837 unique MRNs. We could not use all the MRNs for analyses due to various factors, such as the unavailability of associated demographic data (670 cases) and failure to meet any of the quality control criteria mentioned in On-line Table 3 (1412 cases). Quality control metrics are required because incomplete acquisition, image corruption, erroneous segmentation, DICOM header mislabeling, and misorientation in data present a challenge to automatically detecting nonstenosed canals at all 5 levels. Our final analysis included 1755 MRNs (797 men and 958 women), in which the mean age of men was 49.6 years ( $\sigma=16.92$ years) with a median of 49.5 years. The mean age of women was 48.25 years ( $\sigma=16.25$ years) with a median of 49.0 years. The age ranges for both men and women were between 19 and 81 years. tions had a $100 \%$ detection rate, we attempted a U-Net algorithm for spinal cord segmentations. This model had parameters identical to the U-Net described for disc segmentation. As opposed to the proposed method, the U-Net failed by mis-segmenting the image (Fig 3C). Because such failures are difficult to track using simple quality control criteria (On-line Table 3 ), we espoused the SVM + ERT approach.

\section{Time Improvement}

Human-driven segmentation of a spinal canal takes between 30 and 40 minutes for each image, while machine-generated segmentation takes between 20 and 25 seconds. Thus, machine segmentation is as efficient as expected.

\section{Relationship between Canal Areas and Height, Sex, and Age}

We investigated the relationship between canal areas and demographic factors (height, sex, and age) using a variable-intercept 
Table 2: Age, gender, and height were analyzed using a mixed effects model Canal Area $\sim$ age + gender + height + gender*height $+(1 \mid$ subject). This table represents the Random Effects fit ${ }^{\mathrm{a}}$

\begin{tabular}{lccc}
\hline \multicolumn{4}{c}{ Random Effects } \\
\hline \multicolumn{1}{c}{ Groups } & Name & Variance & SD \\
\hline Group & (Intercept) & 2868 & 53.55 \\
Residual & & 2365 & 48.63 \\
No. of obs & 8775 & No. of subjects & 1755 \\
\hline
\end{tabular}

Note:-obs indicates observers.

a The model fit is summarized. Height is the only variable that is statistically significantly related to canal areas.

Table 3: Age, gender, and height were analyzed using a mixed effects model Canal Area $\sim$ age + gender + height + gender*height + (1 | subject). This table represents the Intercept fit - equivalent to traditional regression ${ }^{\mathrm{a}}$

\begin{tabular}{lcccc}
\hline Fixed Effects & Estimate & Standard Error & $\boldsymbol{T}$ Value & $\operatorname{Pr}(>|\mathbf{t}|)$ \\
\hline (Intercept) & -278.18495 & 43.20594 & -6.439 & 0.00 \\
Age & 0.05158 & 0.08429 & 0.612 & 0.54 \\
Sex & -0.63539 & 62.56533 & -0.01 & 0.10 \\
Height & 8.00079 & 0.66184 & 12.089 & 0.00 \\
Sex:height & -0.46311 & 0.93188 & -0.497 & 0.62 \\
\hline
\end{tabular}

Note: $-\operatorname{Pr}(>|t|)$ indicates $p$-value

${ }^{a}$ The model fit is summarized. Height is the only variable that is statistically significantly related to canal areas.

Table 4: Age, gender, and height were analyzed using a mixed effects model Canal Area $\sim$ age + gender + height + gender*height $+(1 \mid$ subject). This table represents the correlation of fixed effects ${ }^{\mathrm{a}}$

\begin{tabular}{lccrr}
\hline $\begin{array}{l}\text { Correlation of } \\
\text { Fixed Effects }\end{array}$ & (Intercept) & Age & Sex & Height \\
\hline Age & -0.203 & & & \\
Sex & -0.662 & 0.001 & & \\
Height & -0.995 & 0.11 & 0.672 & \\
Sex:height & 0.692 & -0.009 & -0.998 & -0.703 \\
\hline
\end{tabular}

${ }^{a}$ The model fit is summarized. Height is the only variable that is statistically significantly related to canal areas.

mixed linear model. This is because the canal area measurements at L1 through L5 are not independent within subjects. Canal areas were clustered by subject, and a mixed model of the form: Canal Area $\sim$ Age + Sex + Height + Sex $\times$ Height $+(1 \mid$ Subject $)$ is used. We used the $\mathrm{R}$ package lme4 (https://cran.r-project.org/ web/packages/lme4/index.html) to implement the model. Tables 2-4 summarize the model fit and show that height is the only demographic factor statistically significantly correlated with canal areas at L1 through L5.

\section{DISCUSSION}

We describe a computerized pipeline to analyze spinal canal areas with respect to age, sex, and height. Our machine learning algorithm can automatically delineate spinal canals with human-level accuracy in a time-efficient and consistent manner.

We acknowledge that there are certain limitations to the study design. The first is the area of delineation, at the lower levels (L5S1), was chosen to be the thecal sac rather than the full canal. This was because the thecal sac was most directly relevant to nerve compression and thus to stenotic pathology. Because the thecal sac is much more difficult to delineate, especially at lower levels, this can yield partial canal segmentations as seen in Fig $1 F$. A second caveat involves defining asymptomatic images using ICD codes. While these codes are the clinical standard, they can sometimes contain human error. A third limitation of the study was that we could not obtain the demographic data for body mass index and smoking status, both of which could be important covariates in the model.

The high computational burden of training and analysis represented a challenge to the study. More advanced deep learning models could potentially be trained with the use of graphics processing units, which were not available for this study. We would expect such models to perform better than the proposed pipeline. Lack of computational power also held up cross-validation analysis during training. In the future, we intend to obtain additional computational capacity and train more powerful ML models for canal segmentation.

Future work will include shape analysis alongside area measurements. Canal morphology is perhaps as important as canal area to the diagnosis of stenosis. The development of diagnostic shape measures will be an area of focus for future research. Last, in the future, we also hope to investigate how our technique segments spinal canals when severe pathology is present and whether such segmentations can be used for diagnostic purposes.

\section{CONCLUSIONS}

We have used state-of-the-art machine learning technology to establish a normative range of spinal canal areas in the lumbar spine from MR images. We have documented the variation of these areas with respect to age, sex, and height. We believe that these measurements and range are the critical first step to usher in an objective paradigm in the radiologic diagnosis of lumbar stenosis.

Disclosures: Bilwaj Gaonkar-UNRELATED: Employment: University of California Los Angeles, RELATED: Passive stock ownership in Theseus Al, a start up being spun out of University of California Los Angeles, Luke Macyszyn - UNRELATED: Employment: University of California Los Angeles, RELATED: Stock ownership and Board membership in Theseus Al, a start up being spun out of University of California Los Angeles, NIH grant R21EB026665 awardeed as Principal Investigator; We have plans to apply for patents (Planned, Pending or Issued) and wish to have the right to submit patents based on this work in the future.

\section{REFERENCES}

1. Chad DA. Lumbar spinal stenosis. Neurol Clin 2007;25:407-18

2. Genevay S, Atlas SJ. Lumbar spinal stenosis. Best Pract Res Clin Rheumatol 2010;24:253-65 CrossRef Medline

3. Bankier AA, Levine D, Halpern EF, et al. Consensus interpretation in imaging research: is there a better way? Radiology 2010;257:1417 CrossRef Medline

4. Kamper SJ, Apeldoorn AT, Chiarotto A, et al. Multidisciplinary biopsychosocial rehabilitation for chronic low back pain: Cochrane systematic review and meta-analysis. BMJ 2015;350:h444 CrossRef Medline

5. Alpert HR, Hillman BJ. Quality and variability in diagnostic radiology. J Am Coll Radiol 2004;1:127-32

6. Brady A, Laoide RÓ, McCarthy P, et al. Discrepancy and error in radiology: concepts, causes and consequences. Ulster Med J 2012;81: 3-9 
7. Ronneberger O, Fischer P, Brox T. U-Net: convolutional networks for biomedical image segmentation. May 18, 2015. https://arxiv.org/ pdf/1505.04597.pdf. Accessed April 15, 2018

8. Gaonkar B, Beckett J, Villaroman D, et al. Quantitative analysis of neural foramina in the lumbar spine: an imaging informatics and machine learning study. Radiology: Artificial Intelligence 2019;1:180037 CrossRef

9. Ji X, Zheng G, Liu L, et al. Fully automatic localization and segmentation of intervertebral disc from 3D multi-modality MR images by regression forest and CNN. In: Yao J, Vrtovec T, Zheng G, et al, eds. Computational Methods and Clinical Applications for Spine Imaging. CSI 2016. Lecture Notes in Computer Science. Vol 10182. Cham: Springer; 2016. https://link.springer.com/chapter/10.1007/978-3-319-55050-3_9

10. Tu Z, Bai X. Auto-context and its application to high-level vision tasks and 3D brain image segmentation. IEEE Trans Pattern Anal Mach Intell 2010;32:1744-57 CrossRef Medline

11. Peng Z, Zhong J, Wee W, et al. Automated vertebra detection and segmentation from the whole spine MR images. Conf Proc IEEE Eng Med Biol Soc 2005;3:2527-30 Medline

12. Aslan MS, Ali A, Rara $H$, et al. A novel 3D segmentation of vertebral bones from volumetric CT images using graph cuts. In: Bebis, G. et al. eds. Advances in Visual Computing. ISVC 2009. Lecture Notes in Computer Science. Vol. 5876, Berlin: Springer; 2009

13. Zukić D, Vlasák A, Dukatz T, et al. Segmentation of vertebral bodies in MR images. 2012. https://diglib.eg.org/handle/10.2312/PE.VMV. VMV12.135-142. Accessed April 15, 2018
14. Gaonkar B, Xia Y, Villaroman DS, et al. Multi-parameter ensemble learning for automated vertebral body segmentation in heterogeneously acquired clinical MR images. IEEE journal of translational engineering in health and medicine 2017;5:1-2

15. Mirzaalian H, Wels M, Heimann T, et al. Fast and robust 3D vertebra segmentation using statistical shape models. Conf Proc IEEE Eng Med Biol Soc 2013;2013:3379-82 CrossRef Medline

16. Hall FM. The radiology report of the future. Radiology 2009;251:31316 CrossRef Medline

17. Current procedural terminology (CPT). JAMA 1970;212:873-74 Medline

18. Yushkevich PA, Piven J, Hazlett HC, et al. User-guided 3D active contour segmentation of anatomical structures: significantly improved efficiency and reliability. Neuroimage 2006;31:1116-28 CrossRef Medline

19. Dalal N, Triggs B. Histograms of oriented gradients for human detection. https://lear.inrialpes.fr/people/triggs/pubs/Dalal-cvpr05. pdf. Accessed November 10, 2017

20. Kazemi V, Sullivan J. One millisecond face alignment with an ensemble of regression trees. 2014. http://www.csc.kth.se/ vahidk/ papers/KazemiCVPR14.pdf. Accessed November 10, 2017

21. Centers for Disease Control and Prevention, National Center for Health Statistics. International Classification of Diseases, Ninth Revision, Clinical Modification (ICD-9-CM). 2018. https://www. cdc.gov/nchs/icd/icd9cm.htm. Accessed April 15, 2018 